\title{
Comunicação
}

https://orcid.org/0000-0002-9794-6972 D.A.C.F. Ermita

https://orcid.org/0000-0003-1882-6372 R.V. Sepúlveda

https://orcid.org/0000-0002-9084-1879 A.P.B. Borges

[Communication]

\section{Modelo de indução de osteoporose em coelhas - avaliação radiográfica}

[Rabbit osteoporosis induction model - radiographic evaluation]

\author{
F.R. Araújo ${ }^{1}$, F.L. Valente $^{1}$, E.C.C. Reis ${ }^{1}$, D.A.C.F.Ermita ${ }^{1}$, \\ R.V. Sepúlveda ${ }^{2}$, A.P.B. Borges ${ }^{1 *}$
}

${ }^{1}$ Universidade Federal de Viçosa - Viçosa, MG

${ }^{2}$ Universidade Vila Velha - Vila Velha, ES

A osteoporose é uma desordem metabólica do tecido ósseo, com prevalência crescente na população humana, que se caracteriza por desequilíbrio entre a formação e a reabsorção óssea, havendo perda de massa e redução da densidade, acompanhada por danos na microarquitetura, que aumentam os riscos de fraturas (WHO Study group, 1994). Pode ser classificada, segundo a causa, em primária (tipos I e II) e secundária. A osteoporose primária tipo I está associada à deficiência de estrógeno, enquanto a tipo II está associada ao envelhecimento, fase em que ocorre deficiência crônica do cálcio, aumento da atividade do paratormônio e menor formação óssea. A osteoporose secundária é a perda óssea associada a processos inflamatórios, alterações endócrinas e uso de drogas (Korkia, 2002).

O modelo experimental descrito como "padrão ouro" para estudo da osteoporose utiliza ratas ovariectomizadas (Thompson et al., 1995). Entretanto, esse modelo apresenta várias desvantagens, como a incapacidade de alcançar a maturidade esquelética, a falta de um sistema haversiano e a baixa taxa de remodelação no osso cortical. Além disso, o tamanho do osso não permite um defeito ósseo crítico para melhor avaliação de possíveis tratamentos (Veterano, 2013). Nesse contexto, os coelhos vêm sendo utilizados em estudos experimentais por não possuírem tais desvantagens, sendo considerados um modelo experimental mais adequado para avaliação de alguns tratamentos. No modelo com coelhas, é necessária a associação da

Recebido em 13 de maio de 2018

Aceito em 15 de julho de 2019

*Autor para correspondência (corresponding author)

E-mail: andrea@ufv.br ovariectomia com administração de glicocorticoides, entretanto, na literatura, existem diferentes protocolos com resultados variados e nem sempre satisfatórios (Castañeda et al., 2008; Baofeng et al., 2010).

Assim, com o objetivo de estabelecer um modelo de indução de perda óssea em coelhas para o estudo da osteoporose, 18 coelhas foram submetidas à indução da osteoporose pela associação de ovariectomia e administração de glicocorticoide. As normas de conduta para o uso de animais no ensino foram seguidas rigorosamente e o projeto foi aprovado pela Comissão de Ética no Uso de Animais (Ceua) da Universidade Federal de Viçosa, sob protocolo 49/2014.

Para a cirurgia de ovariectomia, os animais foram anestesiados com a associação de quetamina $(12,5 \mathrm{mg} / \mathrm{kg})$ e midazolam $(1 \mathrm{mg} / \mathrm{kg})$, via intramuscular, e mantidos com isoflurano, administrado por via inalatória, com o auxílio de máscara, diluído em oxigênio $100 \%$ em quantidade suficiente para a realização do procedimento. Para o controle da dor no trans e no pós-operatório imediato, foi realizada anestesia epidural lombossacra, com associação de lidocaína $(0,2 \mathrm{ml} / \mathrm{kg})$ e morfina $(0,1 \mathrm{mg} / \mathrm{kg})$. Com os animais anestesiados, foi realizada uma celiotomia mediana retroumbilical de $4 \mathrm{~cm}$. Em seguida, foi realizada incisão na linha alba, com exposição da cavidade abdominal.

$\mathrm{O}$ ovário esquerdo foi exposto e, após a identificação dos vasos ovarianos, nestes foi realizada hemostasia temporária preventiva, com o auxílio de uma pinça hemostática. 
Posteriormente, foi feita a ligadura dos vasos com fio ácido poliglicólico 2-0. O mesmo procedimento foi realizado no ovário direito. Em seguida, foi realizada sutura da linha alba, com fio de náilon 2-0, no padrão Reverdin, bem como do subcutâneo, com Vicryl 3-0, no padrão simples contínuo e, por fim, procedeu-se à sutura da pele com fio de náilon 2-0, no padrão Wolff. A ferida foi, então, protegida com curativo e roupa cirúrgica. A analgesia pós-operatória foi realizada com $10 \mathrm{mg} / \mathrm{kg}$ de tramadol, via subcutânea, a cada oito horas, durante cinco dias. A administração do glicocorticoide foi iniciada duas semanas após a ovariectomia, para que não interferisse na cicatrização da ferida cirúrgica. Foram administrados, por via intramuscular, na dose de $1 \mathrm{mg} / \mathrm{kg}$ de metilprednisolona, em dias intercalados, durante duas semanas.

Imediatamente antes da ovariectomia e seis semanas após o término do glicocorticoide, foram realizadas radiografias do olecrano direito na posição mediolateral, sendo a técnica radiográfica padronizada, com quilovoltagem de $45 \mathrm{kv}$, miliamperagem de $100 \mathrm{~mA}$, tempo de 0,07 segundos e distância foco-filme de $95 \mathrm{~cm}$. Todas as radiografias foram tomadas com um penetrômetro de alumínio (Fig. 1A), constituído por cinco degraus, com espessura de $0,3 \mathrm{~mm}$ entre cada um deles, posicionado próximo ao olecrano. As imagens obtidas foram analisadas no programa Image $^{\circledR}$, para determinação da densidade óssea em milímetros de alumínio (mm $\mathrm{Al})$, da seguinte forma: foi selecionada uma área de 25 x 200 pixels, com auxílio da ferramenta de seleção retangular, em cada degrau do penetrômetro, sendo, então, aferida a tonalidade média de cinzas. Os valores obtidos foram armazenados sob a forma de tabelas, e, de posse da informação do nível de cinza e de sua respectiva espessura, foi obtida uma regressão linear, no programa Excel, em que a coordenada $\mathrm{X}$ é a densidade em $\mathrm{mm}$ de $\mathrm{Al}$, e $\mathrm{Y}$ o nível de cinza (Fig. 1B).

Posteriormente, a ferramenta de seleção de área elíptica foi utilizada para obtenção do valor do grau de cinza da região do defeito no olecrano, no tamanho de 50 x 50 pixels. Esses valores foram substituídos na fórmula obtida por meio do penetrômetro, fornecendo a densidade em $\mathrm{mm}$ de Al da região desejada. A densidade radiográfica da indução foi analisada pelo teste tpareado, no programa SigmaPlot 11.0, considerando-se nível de significância de $5 \%$.
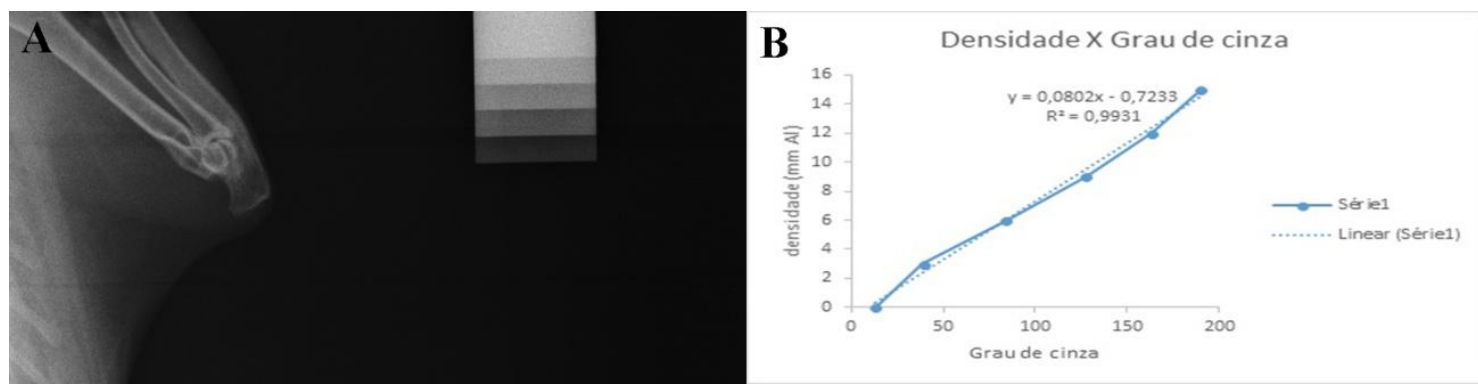

Figura 1. (A) Radiografia mediolateral do olecrano de coelho, ao lado do penetrômetro, após 42 dias do término da aplicação do glicocorticoide. (B) Exemplo do gráfico e da função linear de um animal, obtidos a partir do grau de cinza dos degraus do penetrômetro. O modelo de indução utilizado no presente trabalho foi baseado em estudos realizados por Castañeda et al. (2008), os quais observaram que, para a indução da osteoporose em coelhas, apenas a ovariectomia não é suficiente, sendo necessária a associação com a aplicação de glicocorticoide. A densidade radiográfica média dos animais antes do processo de indução da osteoporose, ou seja, antes da ovariectomia e do glicocorticoide, foi igual a 2,305 $\pm 0,488 \mathrm{~mm}$ Al. Em contrapartida, 42 dias após o término da aplicação do glicocorticoide, a densidade radiográfica foi de $1,575 \pm 0,174 \mathrm{~mm} \mathrm{Al}$, representando uma queda significativa $(\mathrm{P}<0,05)$ da densidade ao longo do tempo, o que comprova que o protocolo adotado para indução da osteoporose foi capaz de desencadear perda óssea nesses animais, conforme esperado.

A deficiência do estrógeno, associada à administração de glicocorticoide, foi capaz de promover um desequilíbrio entre a reabsorção e a formação óssea, possivelmente por meio do aumento da produção de RANKL, da diminuição de OPG e da diferenciação e função dos 
osteoblastos, conforme descrito por Khosla et al. (2012).

A redução da densidade mineral óssea em termos percentuais foi de aproximadamente $31,6 \%$, semelhante ao resultado encontrado por Baofeng et al. (2010), que observaram uma redução de $36 \%$ na densidade dos animais que receberam corticoide associado à ovariectomia. É importante ressaltar que a osteoporose humana é definida como uma redução na DMO de mais de 2,5 desvios-padrão (WHO Study group, 1994), o que seria equivalente a aproximadamente $25 \%$, indicando que a perda óssea dos animais, no presente estudo, foi suficiente para ser caracterizada como osteoporose.

A Organização Mundial da Saúde (WHO Study group, 1994) também definiu que a densitometria óssea é a ferramenta padrão para o diagnóstico da osteoporose, entretanto, na medicina veterinária, existe o inconveniente de que o paciente a ser examinado deve permanecer, no mínimo, de 10 a 15 minutos imóvel, sendo necessária a utilização de protocolos anestésicos para a realização do exame (Toll et al., 1994). Por outro lado, a densitometria óptica radiográfica, adotada no presente trabalho, possui vantagens, como baixo custo, rapidez, precisão e simplicidade de execução, quando comparada às outras técnicas utilizadas, e vem sendo amplamente utilizada no diagnóstico e acompanhamento das afecções ósseas, com boa confiabilidade (Prado Filho e Sterman, 2004; Muramoto et al., 2005).

Com isso, pode-se afirmar que o protocolo adotado foi suficiente para provocar uma perda óssea significativa, caracterizando um modelo válido para o estudo de novos tratamentos para a osteoporose.

\begin{abstract}
In order to establish a bone loss induction model in rabbits to study osteoporosis, 18 rabbits underwent ovariectomy and received methylprednisolone $1 \mathrm{mg} / \mathrm{kg}$ intramuscularly on alternate days for two weeks. Immediately before ovariectomy up to 42 days after methylprednisolone administration, radiographs of the right olecranon were taken in the mediolateral position to evaluate the radiographic optical density. Before the induction of osteoporosis, rabbits presented mean values of radiographic density of $2.305 \mathrm{~mm}$ Al and at 42 days of $1.575 \mathrm{~mm}$ Al. The values obtained were submitted to ANOVA for repeated measures that revealed a significant drop $(P<0.001)$ in the density over time, proving that the induction was able to trigger bone loss of these animals. With this, it can be affirmed that the adopted protocol was enough to provoke a significant bone loss, characterizing a valid model for the study of new treatments for osteoporosis.
\end{abstract}

Keyword: osteopenia, bone mineral density, regenerative medicine

\section{AGRADECIMENTOS}

Coordenação de Aperfeiçoamento de Pessoal de Nível Superior (Capes), Conselho Nacional de Desenvolvimento Científico e Tecnológico (CNPq) e Fundação de Amparo à Pesquisa do Estado de Minas Gerais (Fapemig).

\section{REFERÊNCIAS}

BAOFENG, L.; ZHI, Y.; BEI, C. et al. Characterization of a rabbit osteoporosis model induced by ovariectomy and glucocorticoid. Acta Orthop., v.81, p.396-401, 2010.

CASTAÑEDA, S.; CALVO, E.; LARGO, R. et al. Characterization of a new experimental model of osteoporosis in rabbits. J. Bone Mineral Metabol., v.26, p.53-59, 2008.
KHOSLA, S.; OURSLER, M.J.; MONROE, D.G. Estrogen and the Skeleton. Trends Endocrinol. Metabol., v.23, p.576-581, 2012.

KORKIA, P. Osteoporosis : process , prevention and treatment. J. Lecturer Sport Excerc. Sci., v.6, p.156-169, 2002.

MURAMOTO, C.; STERMAN, F.A.; PINTO, A.C.B.C. Estabelecimento de valores de densidade mineral óssea (DMO) das regiões metafisária e diafisária do rádio em cães da raça Poodle por meio da Densitometria Óptica Radiográfica. Braz. J. Vet. Res. Anim. Sci., v.42, p.89-97, 2005. 
PRADO FILHO, J.R.C.; STERMAN, F.A. Avaliação da densidade mineral óssea em potros da raça Puro Sangue Inglês em início de treinamento. Braz. J. Vet. Res. Anim. Sci., v.41, p.384-388, 2004.

THOMPSON, D.D.; SIMMONS, H.; PIRIE, C. et al. FDA guidelines and animal models for osteoporosis. Bone, v.17, p.S125-S133, 1995.

TOLL, P.W.; GROSS, K.L.; BERRYHILL, S.A. et al. Usefulness of dual energy X-ray absorptiometry for body composition measurement in adult dogs. J. Nutr., v.124, p.2601S-2603S, 1994.
VETERANO, W. The laboratory rat as an animal model for osteoporosis vertentes a abordar. Am. Assoc. Lab. Anim. Sci., v.58, p.424-430, 2013.

WHO Stduy group. Assessment of fracture risk and its application to screening for postmenopausal osteoporosis. WHO Techn. Rep. Ser., v.843, p.1-130, 1994 